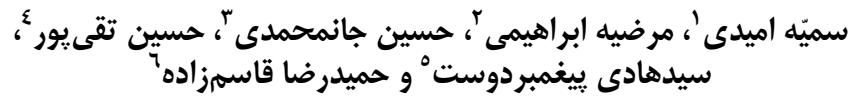

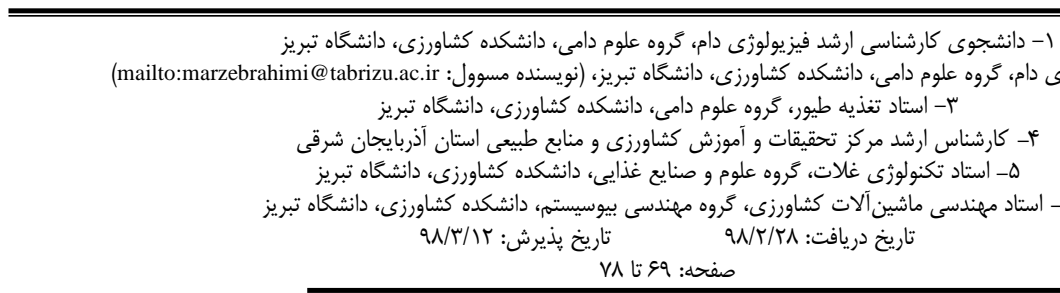

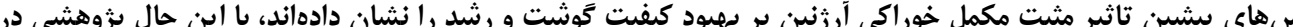

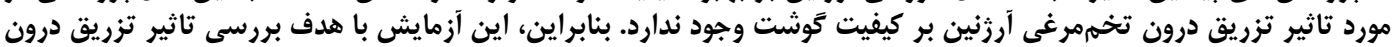

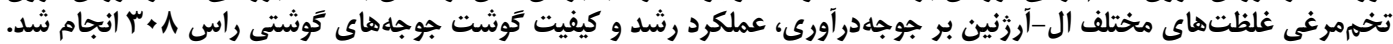

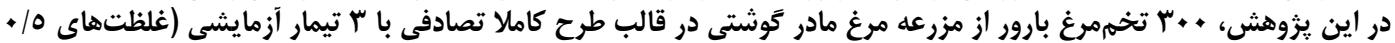

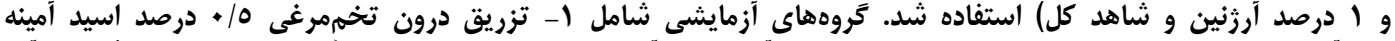

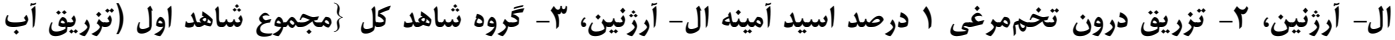

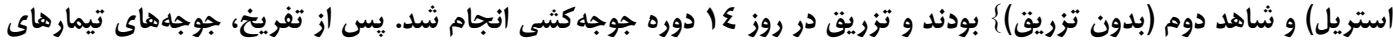

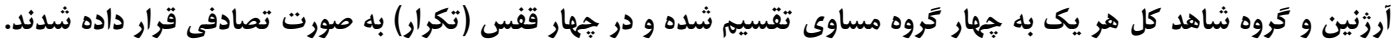

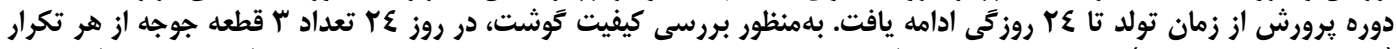

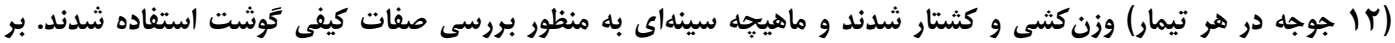

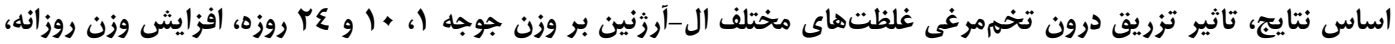

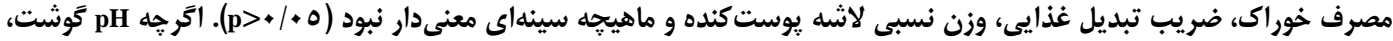

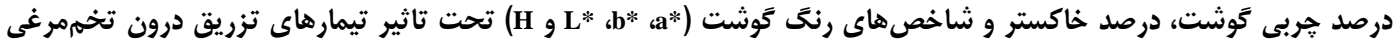

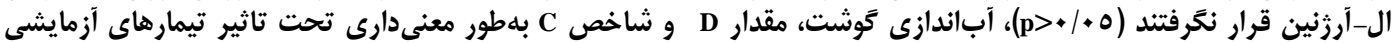

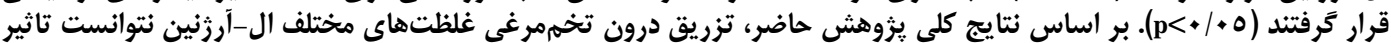

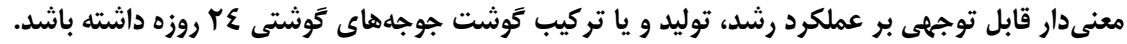

$$
\text { وازههاى كليدى: ال-آرزنين، تزريق درون تخهمرغى، كيفيت كَوشت، جوجه كَوشتى }
$$

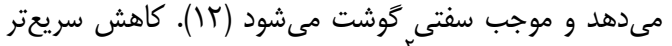

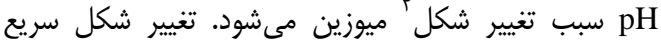

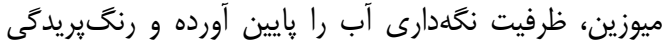

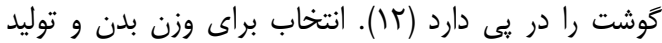

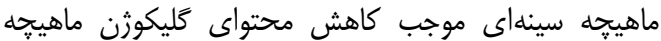

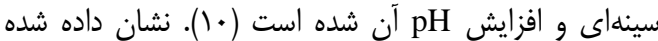

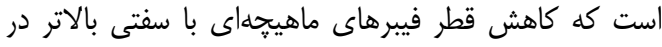

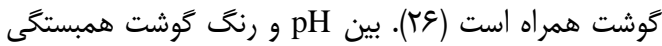

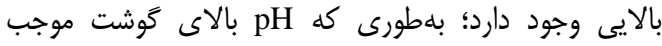

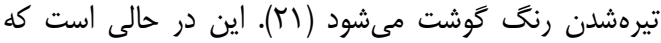

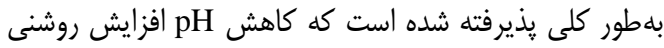

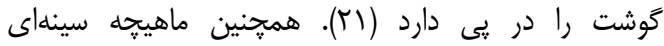

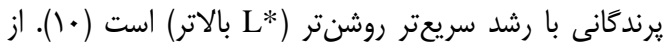

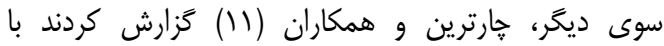

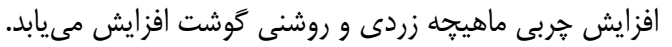

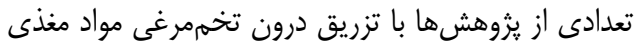

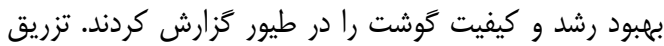

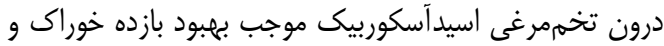

مقدمه

بهدليل اين كه در روند اصلاح زنتيكى جوجه كوشتى، تنها

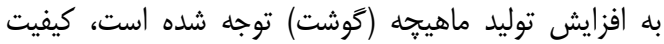

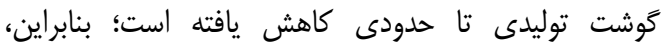

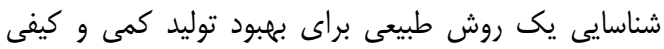

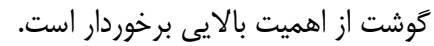

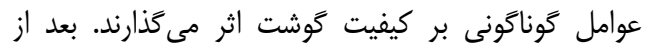

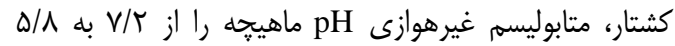

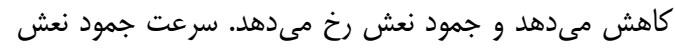

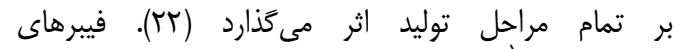

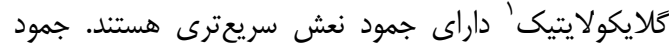

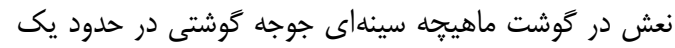

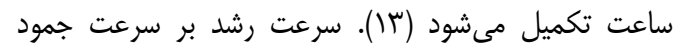

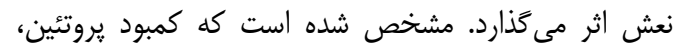

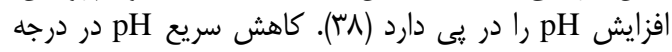

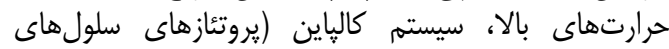

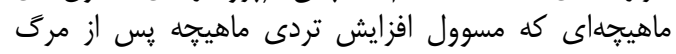

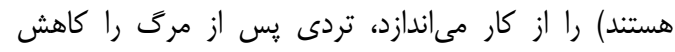




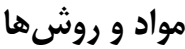

اين يزوهش با هدف بررسى تاثير تزريق درون تخممرغى

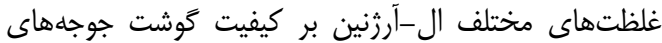

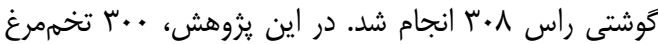

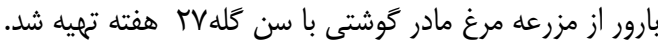

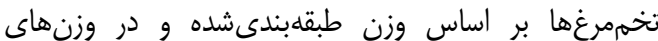

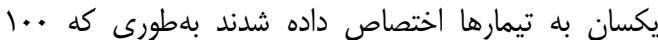

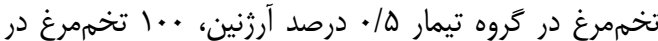

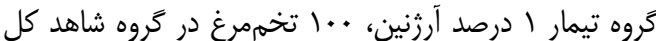

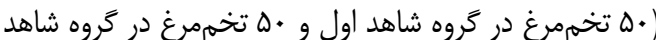

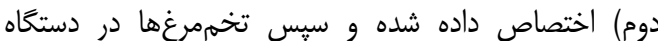

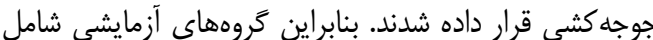

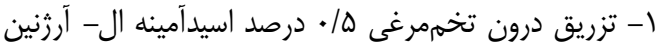
(ه ميلى

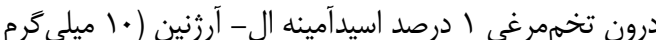

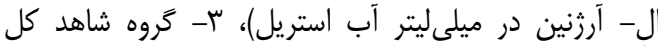

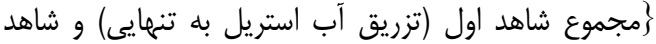

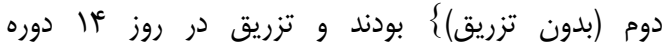

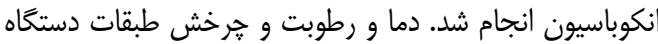

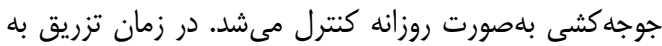

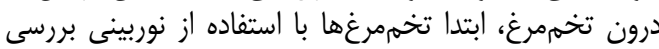

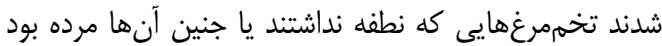

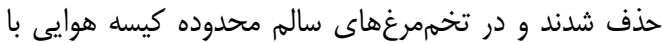

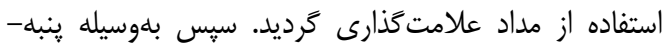

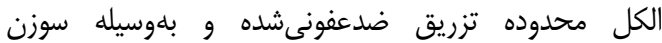

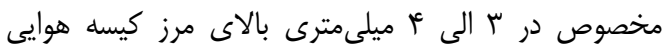

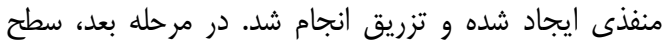

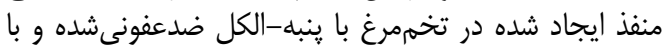

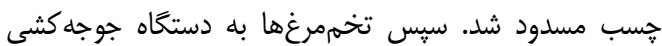

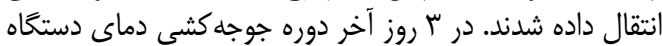

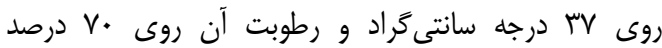

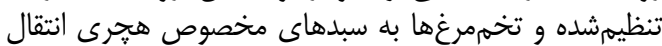

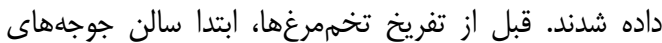

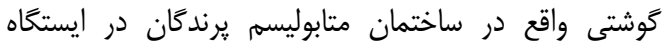

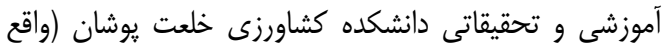

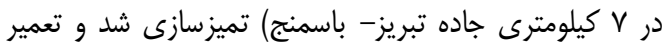

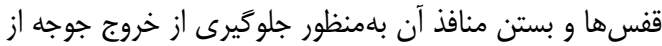

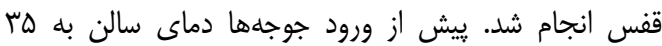
درجهسانتى

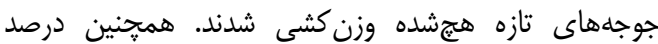

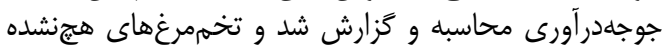

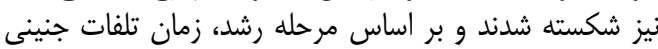

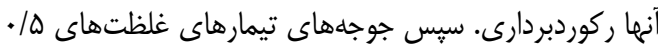

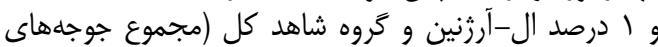

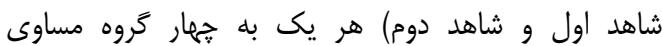

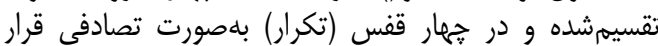

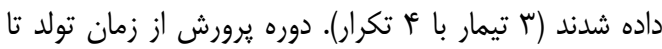

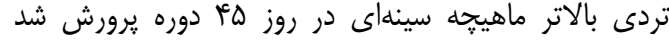

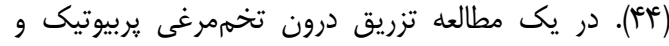

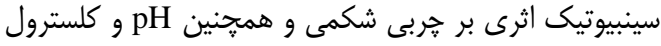

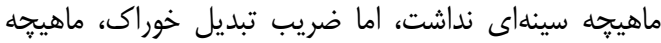

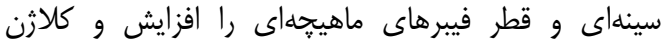
ماهيجه و توليد لاشه را كاهش يافينه

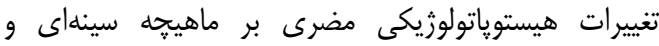

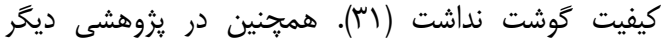

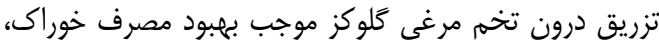

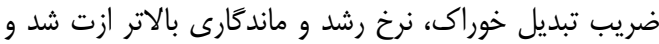

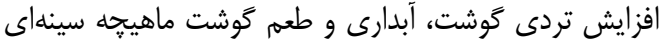

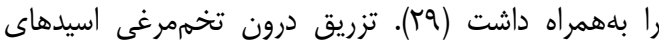

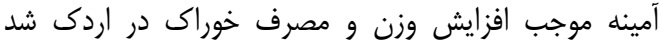

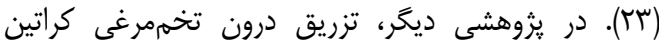

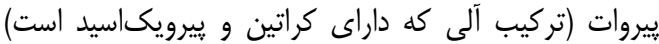

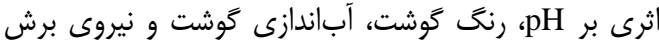

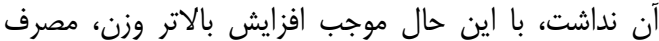

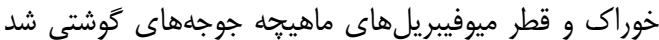

آرزنين يك اسيدآمينه ضرورى در طيور است كه بلهصورت

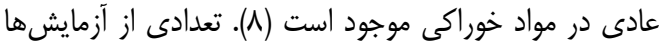

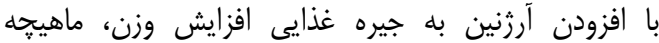

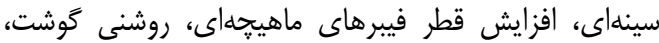

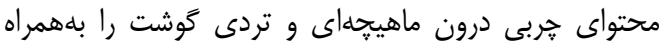

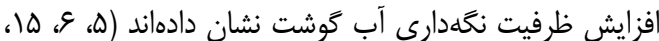

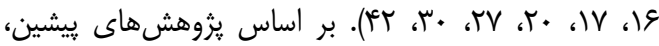

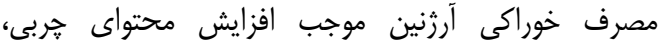

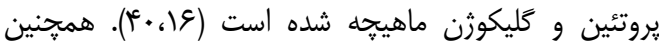

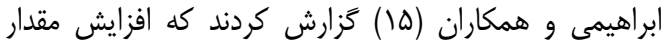

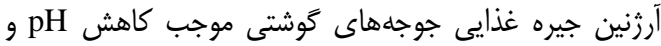

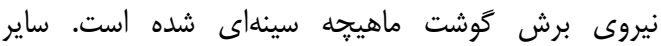

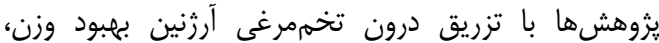

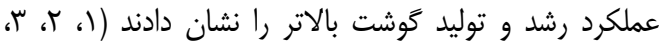

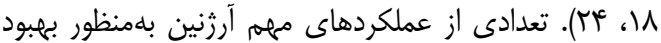

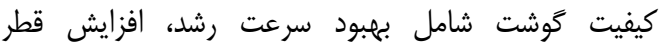

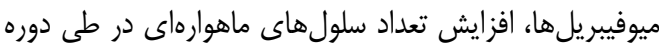

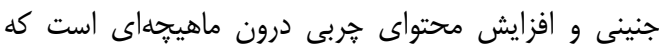

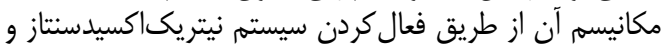

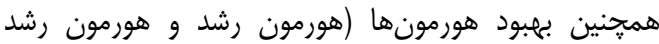

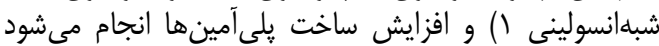

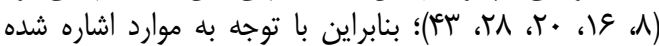

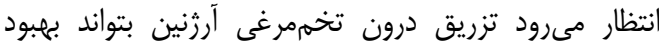

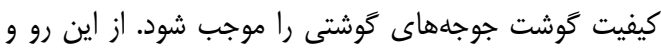

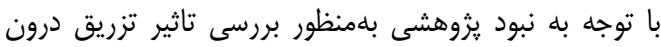

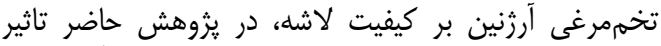

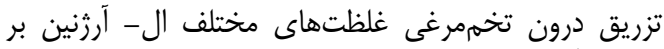

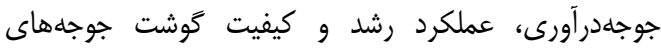
كوشتى بررسى شد. 
جوجهدرآورى با هر دو شيوه آزمون فيشر و رويه GLM

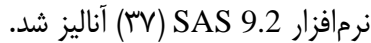
مدل آمارى يزوهش حاضر به شرح ذيل بود:

$\mathrm{Y}_{\mathrm{ijk}}=\mu+\mathrm{T}_{\mathrm{i}}+\mathrm{S}_{\mathrm{j}}+\mathrm{b}\left(\mathrm{Y}_{\mathrm{ijk}}-\overline{\mathrm{Y}}_{000}\right)+\mathrm{e}_{\mathrm{ijk}}$

i=1, 2, 3 (تيماره)

j=1, 2 (اثر جنسارها)

$\mathrm{k}=1-12$

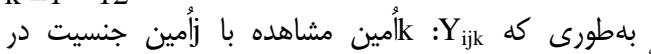

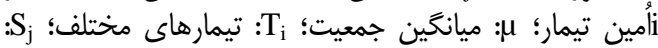

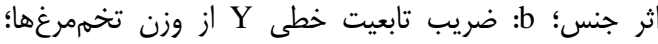
Y

\section{نتايج و بحث

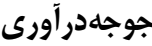

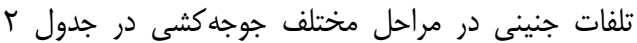

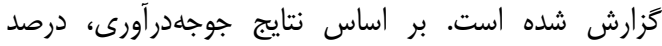

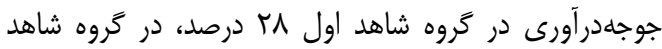

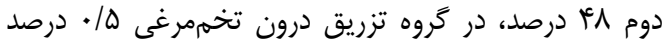

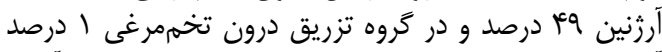

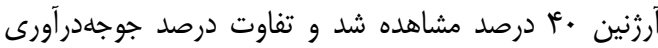

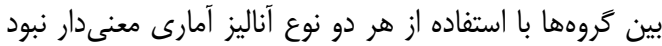

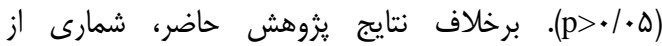

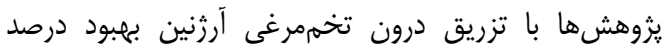

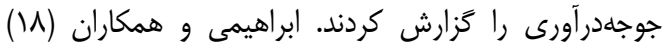

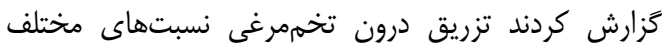

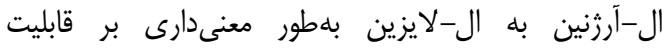

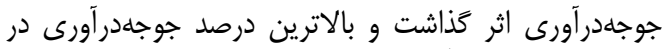

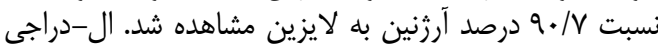

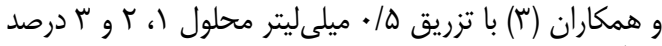

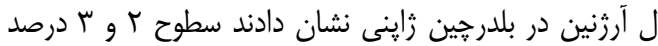

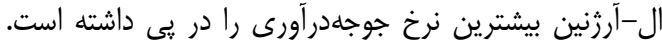

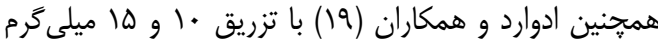

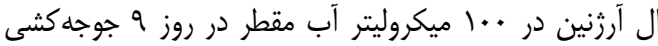

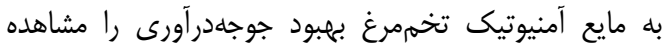

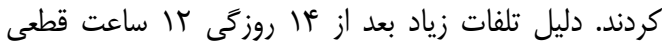

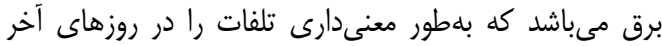

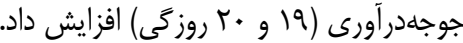

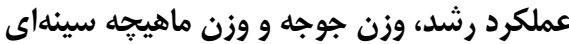

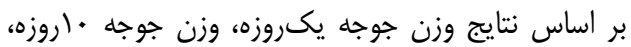

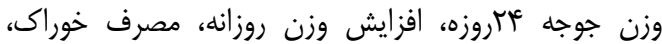

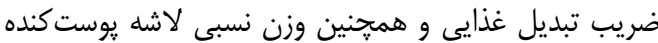

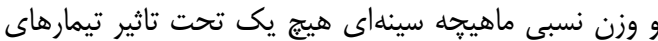

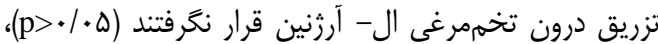

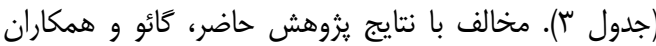

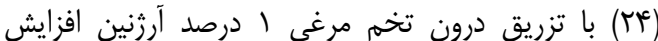

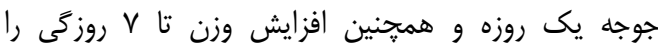

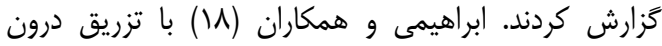

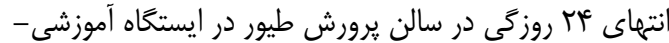

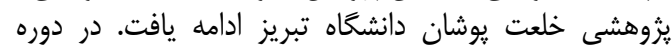

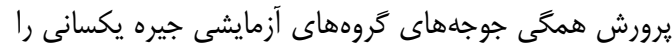

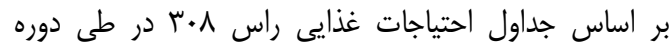

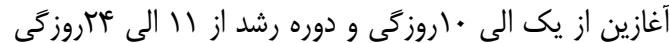

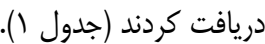

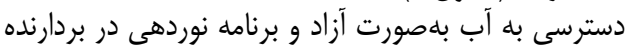

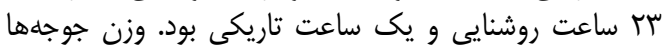

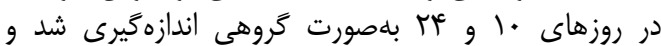

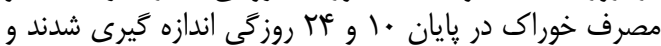

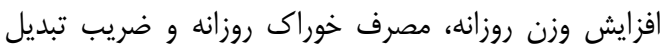

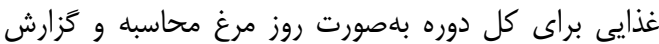

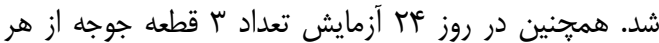

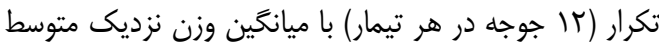

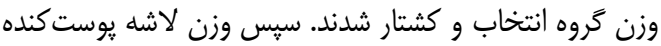

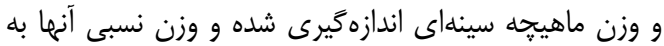

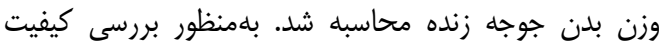

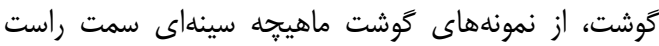

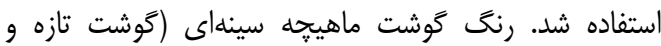

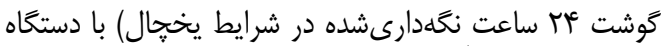

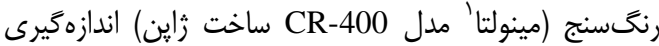

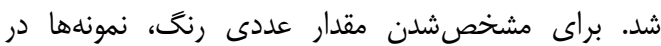

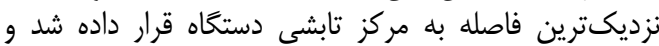
نتايج بلهصورت

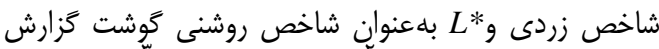

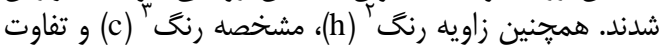

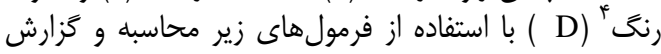
$\mathrm{h}=\arctan \left(\mathrm{b}^{*} / \mathrm{a} *\right)$

$\mathrm{c}=\left(\mathrm{a}^{* 2}+\mathrm{b}^{* 2}\right)^{1 / 2}=\left[\left(\mathrm{L}^{*} \mathrm{~L}_{1}-\mathrm{L}_{2}\right)^{2}+\left(\mathrm{a}^{*}{ }_{1}-\mathrm{a}_{2}\right)^{2}+\left(\mathrm{b} *_{1}-\mathrm{b}_{2}\right)^{2}\right]^{1 / 2}$

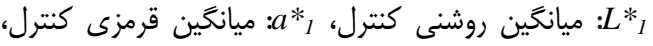

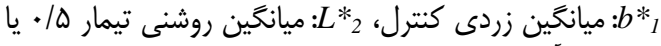

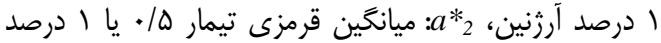

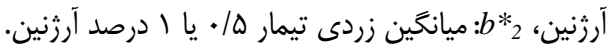

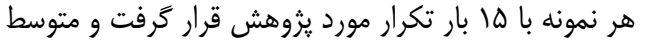

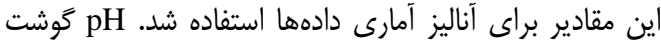

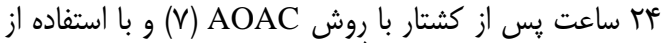

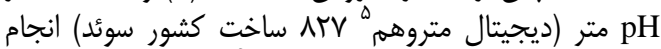

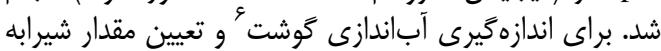

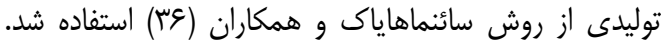

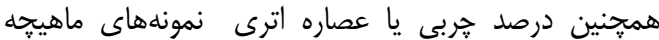

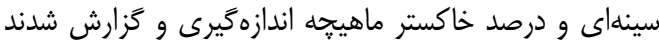

در קِيان دادهها با استفاده از رويه GLM نرمافزار

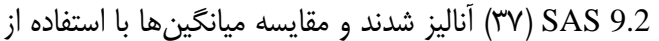

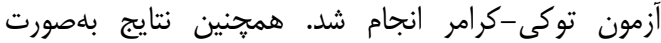

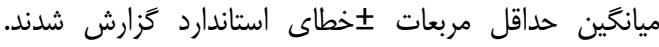

4- Colour differ 5- Metrohm

1- Minolta 2- Hue angle 3-Chroma

6- Drip loss 7- Ether Extract 
بالاى آرزنين موجب بهبود عملكرد رشد، وزن جوجههاى

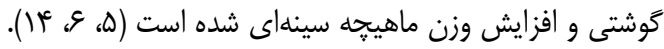

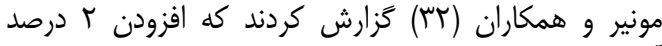

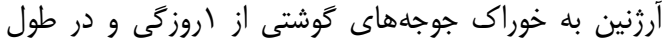

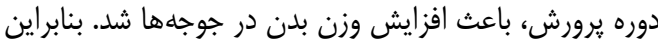

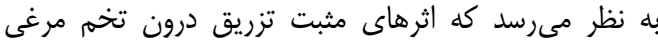

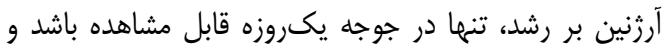

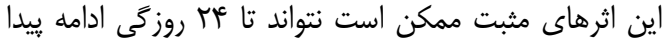

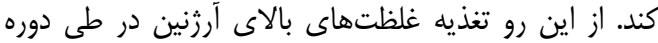
يرورش مىتواند از ادامه روند رشد حمايت بيشترى كند.
تخمرغیى نسبتهاى مختلف ال-آرزنين به ال-لايزين

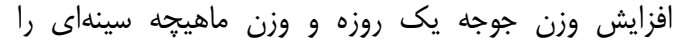

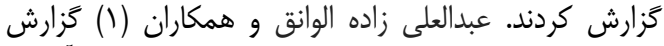

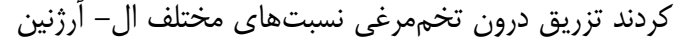

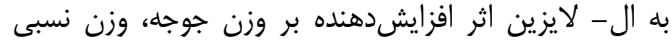

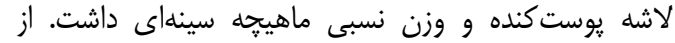

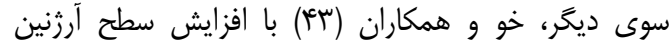

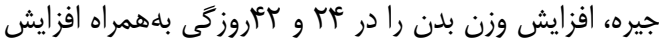

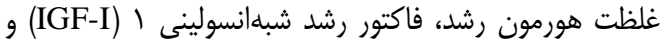

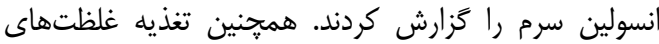

جدول ا- اجزاء و تركيب مواد مغذى جيره آغازين و رشد جوجههاى گَوشتى (بر اساس ماده خشك) Table 1. Ingredient composition and nutrient concentrations of the starter and grower diets (on a dry matter basis)

\begin{tabular}{|c|c|c|}
\hline 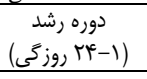 & 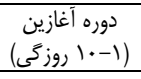 & تركيب جيره \\
\hline$\Delta N / \bar{G}$ & $\Delta V / \Delta$ & ذرت \\
\hline$r / \Delta$ & 1 & 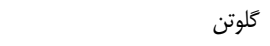 \\
\hline$r 9 / 1$ & rN/G & 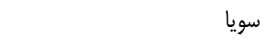 \\
\hline f & $r / \Delta$ & يودر ماهى \\
\hline$\cdot / 1$ & $1 / 4$ & روغن \\
\hline $1 / v \Delta$ & r & دى كلسيمفسفات \\
\hline $1 / 1$ & $1 / r$ & كربنات كلسيم \\
\hline.$/ 1 V$ & $\cdot / 1 \wedge$ & نمى \\
\hline- & .1 .9 & جوششيرين \\
\hline.$/ \Delta$ &.$/ 8$ & مكمل ويتامين و مواد معدنى \\
\hline •/ & 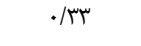 & متيونين \\
\hline$\cdot / T V$ & $\cdot / T \Delta$ & ليزين \\
\hline- &.$/ 1$ & ويتامين D3 \\
\hline- &.$/ 1$ & E ويتامين E \\
\hline- & $.1 \cdot 1$ & كولين كلر ايد \\
\hline$\cdot 1 \cdot 0$ & $\cdot 1 \cdot 0$ & ضدكو كسيديوز \\
\hline \multirow[t]{2}{*}{$.1 \cdot \Delta$} & .1 .0 & ضدقارج \\
\hline & & تركيب مواد مغذى جيره بايه \\
\hline$r \cdot \Delta \cdot$ & $r \cdots$ & انرزى (كيلو كالرى در كيلوگرم) \\
\hline $19 / \Delta$ & $r M / \Delta$ & يروتئين كل (درصد) \\
\hline $1 / 1$ & 1/r & ليزين (درصد) \\
\hline$\cdot / \Delta T$ & $\cdot / \Delta \Delta$ & متيونين (درصد) \\
\hline$\cdot / \notin \Delta$ & $\cdot / \Delta$ & فسفر قابل دسترس (درصد) \\
\hline.$/ 9$ & 1 & كلسيهم (درصد) \\
\hline
\end{tabular}


Table 2. The effect of in ovo injection of different levels of L-arginine on hatchability

جدول r- اثر تزريق درون تخممرغى سطوح مختلف ال-آرزنين بر جوجهدرآورى

\begin{tabular}{|c|c|c|c|c|c|}
\hline \multicolumn{6}{|c|}{ وضعيتهاى مختلف } \\
\hline كل & بدون نطفه & زنده & تلفات بعد از ؟1 روزكى & تلفات قبل از fl أروزگى & تيمار \\
\hline & V & If & TE & $r$ & \\
\hline a. & 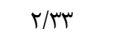 & $r / 9 V$ & $N / 9 V$ & $1 / \cdot$. & \\
\hline & $\mid f /$. & $r N / \cdot$. & $\Delta T / . \cdot$ & $8 / .$. & شاهد اول \\
\hline & איז/سז & $11 / . r$ & $r I / q V$ & $9 /{ }^{\prime} \wedge$ & \\
\hline & $\Delta$ & re & iv & r & \\
\hline $\begin{array}{l}\text { D. } \\
\varepsilon<\mid q \gamma\end{array}$ & $1 / 9 \mathrm{~V}$ & $N /$. & $\Delta / \overline{Q V}$ & איז/1 & \\
\hline & 1.1. & $\mathrm{HN} / .$. & $m F / .$. & $N /$. & شاهد دوم \\
\hline & 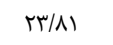 & $1 N / 9$. & $\mid f / N V$ & $1 \pi / \Delta$ & \\
\hline & r & 19 & rv & 1. & \\
\hline 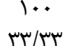 & " سז/1 & س & س & 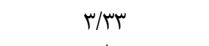 & تزريق ه/• درصد \\
\hline זוז/אז & $r /$. & $+q / .$. & $\mathrm{r} / /$. & $1 . \%$ & آرثنين \\
\hline & $19 / \cdot \Delta$ & 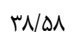 & $r \cdot / \Lambda \mu$ & TI/Ta & \\
\hline & $\Delta$ & $r$. & r. & 10 & \\
\hline 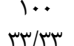 & $1 / 9 V$ & سז/Tו & س ( & $\Delta / .$. & تزريق ادرصد \\
\hline זוז/זr & $\Delta / . \cdot$ & $f . / .$. & $r \cdot 1 .$. & $\mid Q / \cdot$. & آرزنين \\
\hline & $r / \Lambda)$ & $\mu / \Delta$. & 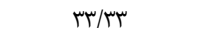 & Es/M & \\
\hline$\mu .$. & rI & $I T V$ & Ir. & r & \\
\hline $1 \ldots 1$. & $\mathrm{V} / \cdot$. & Tr/Tr & $f \cdot 1$. & $1 . / 9 V$ & كل \\
\hline
\end{tabular}

در هر ستون دادها به ترتيب از بالا به پايين شامل تعداد، درصد كل، درصد در هر تيمار و درصد در هر ستون مىباشد.

آباندازى ماهيجه سينهاى

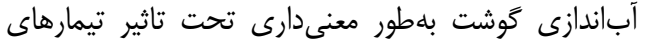

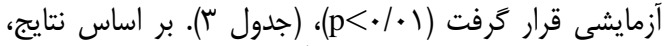

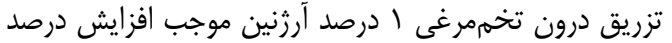

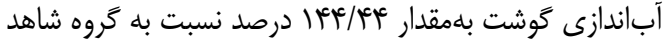

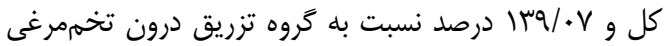

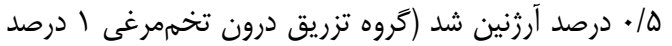

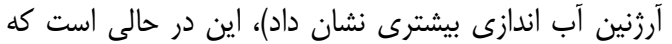

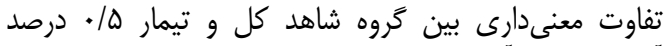

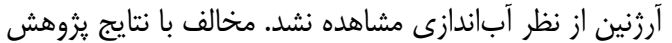

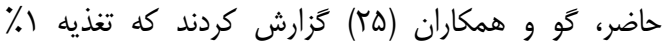

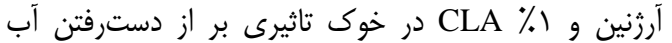

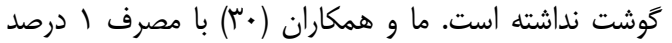

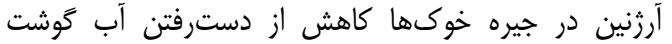

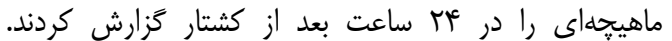
كزارش شده است كه كاهش سريع pH موجب تغيير شكل

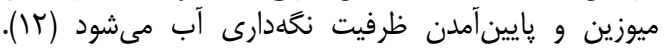

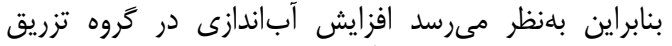

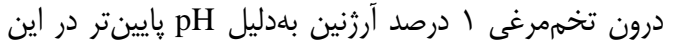

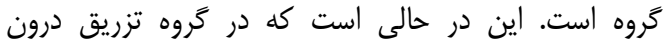

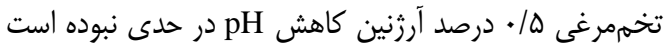

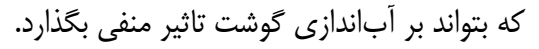
جربى و خاكستر ماهيجه سينهاى

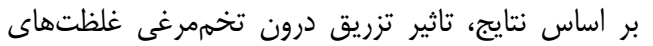

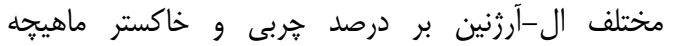

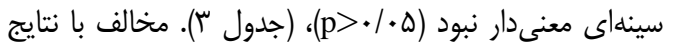

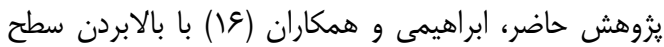

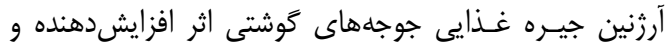

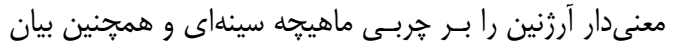

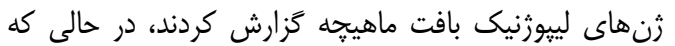

ماهيجٌه سينهاى

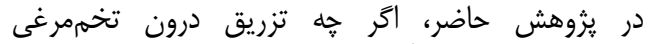

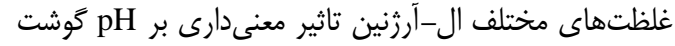

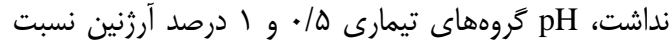

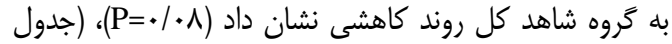

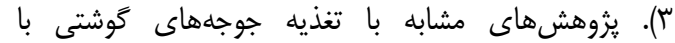

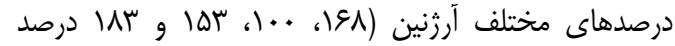

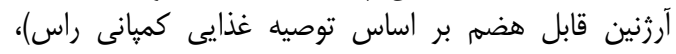

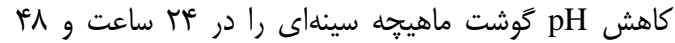

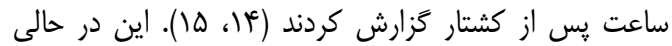

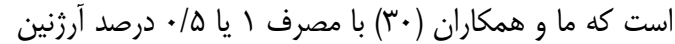

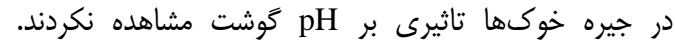

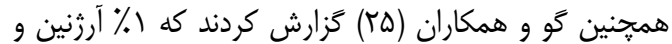

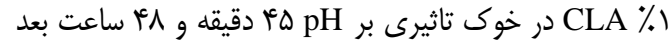

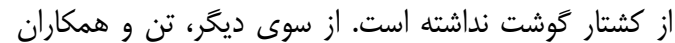

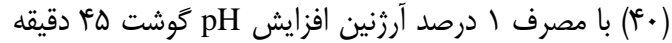

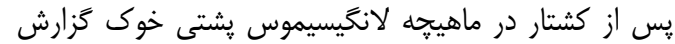

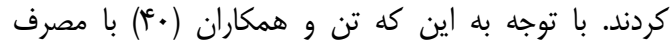

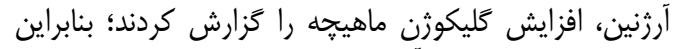

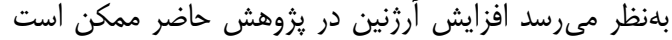

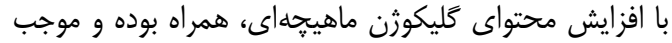

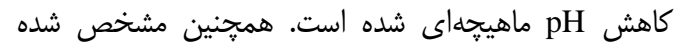

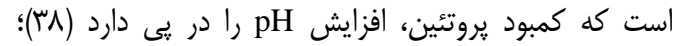

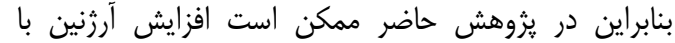

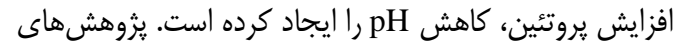

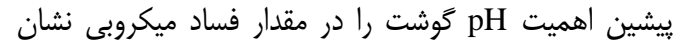

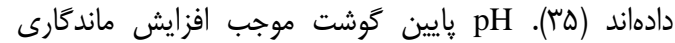

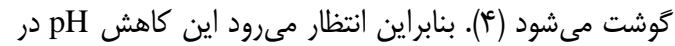

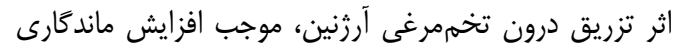
كوشت شود. 
نبود تاثير معنىدار تزريق غلظتهاى مخته مختلف ال-آرزنين

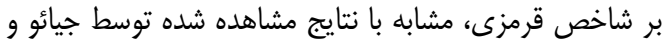

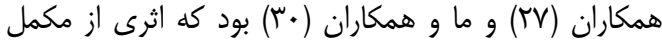

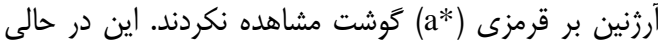

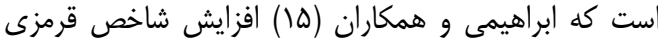
كَوشت ماهيحه سينهاى را با افزايش آرزنين جيره كزارش كردند.

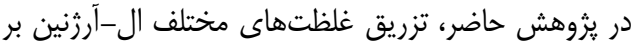

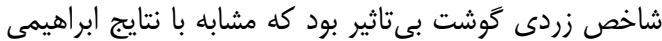

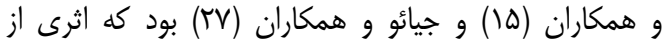
افزودن سطح آرزنين خوراكى بر شاخص زئر زردى كَّت

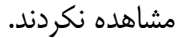

از سوى ديخر، روشنى كوشت تحت تاثير تزريق

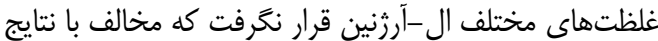

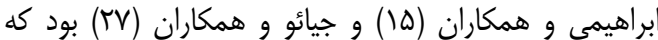

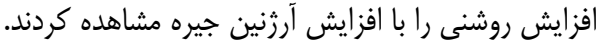

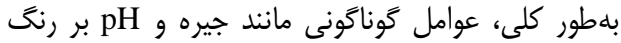

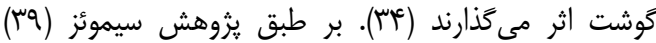

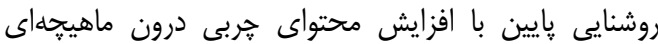

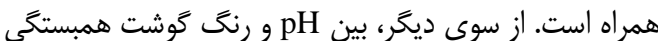

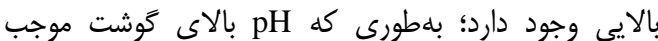

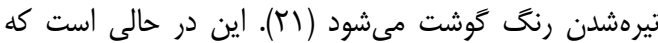

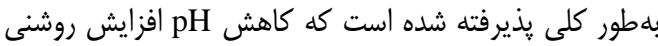

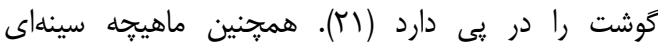

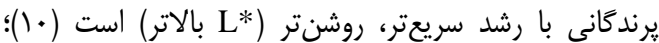

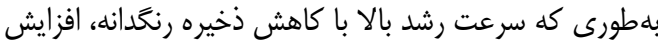

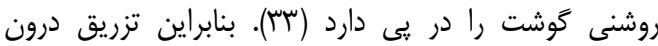

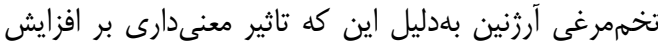

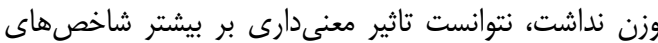
رنخ كَشت بخذارد.

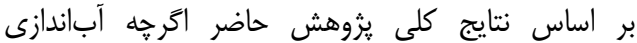

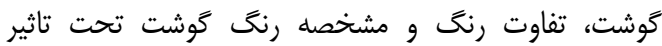

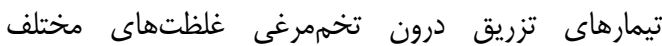

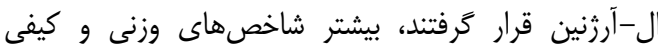

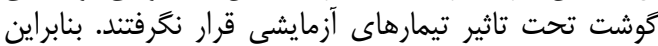

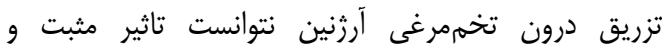

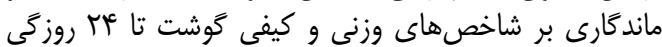

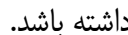

تشكر و قدر دانى بدينوسيله از همكارى جناب آقاى دانى دكتر غالامعلى مقدم،

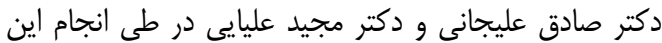

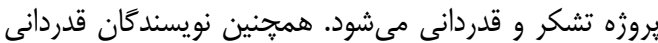

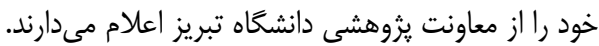

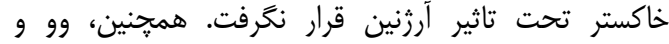

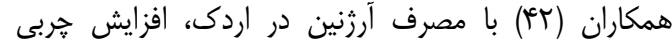

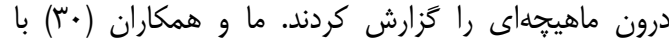

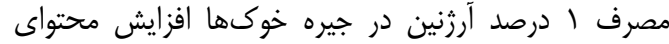

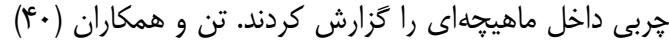

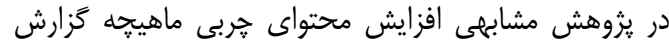

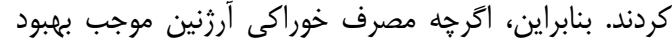

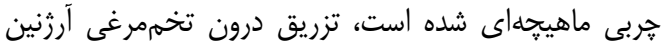

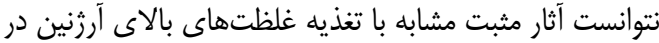
طى دوره رشد را داشته باشد.

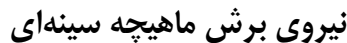
نتايج آزمايش حاضر نشان دادئ داد تاثير تزريق غلظتهاي

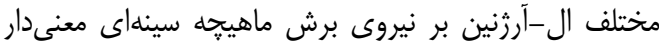

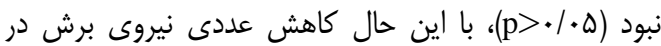

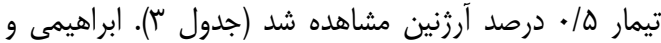

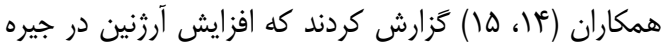

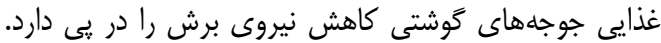

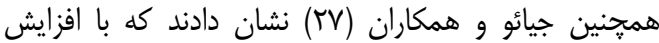

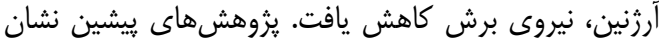

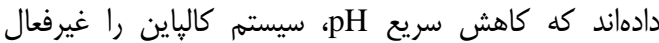

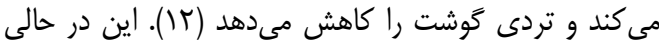

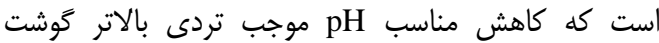

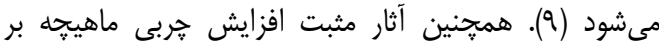

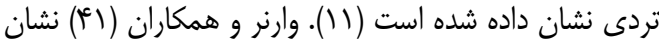

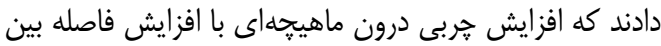

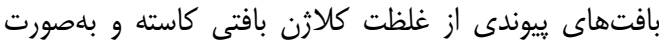

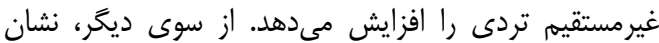

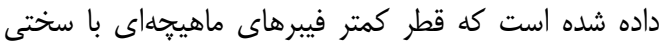

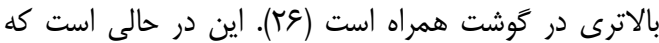

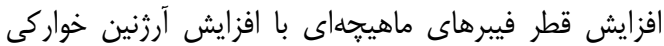

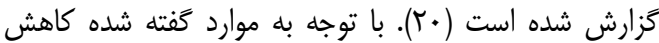

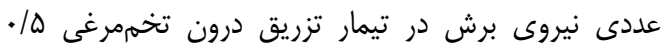

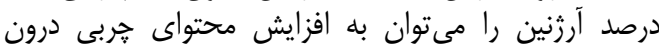

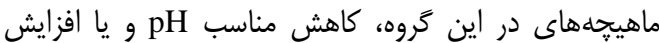
احتمالى قطر ميوفيبرها در اين كروه مره مربوط دانست. رنتى توشت

تيمارهاى آزمايشى تاثير معنىدارى بر شاخصهاى رنى رنى

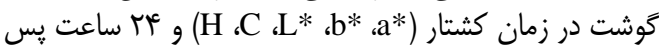

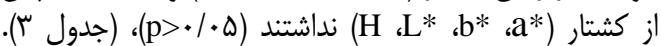

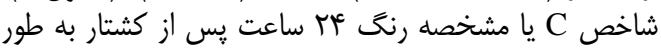

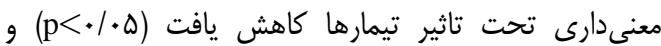

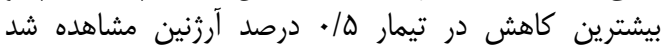

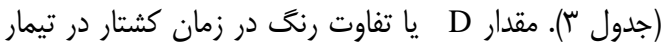

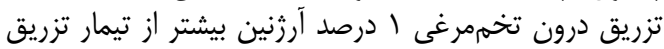

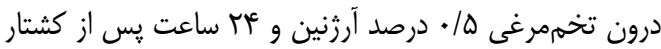

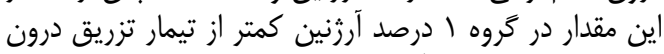
تخممرغى ه/ • درصد آرزثنين بود (جدول آرنين). 
جدول ب- تاثير تزريق درون تخممرغى غلظتهاى مختلف ال-آرزنين بر عملكرد رشد، وزن بدن و كميت و كيفيت كَوشت ماهيحه.

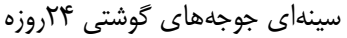

Table 3. The effect of different levels of L-arginine in ovo injection on growth performance, body weight and quantity and quality of breast meat in 24-day-old broiler chickens

\begin{tabular}{|c|c|c|c|c|}
\hline \multirow[b]{2}{*}{ P-value } & \multicolumn{3}{|c|}{ تزريق درون تخهمرغى غلظتهاى مختلف ال-آرزنين } & \multirow[b]{2}{*}{ صفات مورد اندازهگيرى } \\
\hline & تزريق ا درصد آررثنين & تزريق ه/• درصد آرثنين & 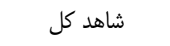 & \\
\hline.$/ 48$ & 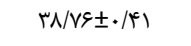 & $r q / 9 \vee \pm \cdot / r \Lambda$ & $r q / \mu I \pm . / 4 r$ & وزن جوجه يكروزه (گرم) \\
\hline$\cdot / 90$ & $r / 9 / 4 \Delta \pm r \cdot / \Lambda \Lambda$ & $r Y G / V V \pm r \cdot / \Lambda \Lambda$ & $r / \Lambda / \Delta \cdot \pm r \cdot / \Lambda \Lambda$ & وزن جوجه • اروزه (كرم) \\
\hline . & $\Lambda \mu \cdot / \cdot r \pm r \varphi / \wedge q$ & $\Lambda T I / 4 Y \pm Y Y / \Lambda q$ & $\Lambda \Delta F / G F \pm r F / \wedge q$ & وزن جوجه أبروزه (كرم) \\
\hline . $/ 4 t$ & $r \mid / \Delta V \pm 1 / \cdot \varphi$ & $r \mid / \wedge \varepsilon \pm 1 / \cdot \varphi$ & $r r / f V \pm 1 / \cdot 9$ & افزايش وزن روزانه FF_ اروزگى (كرم) \\
\hline$\cdot 1 \cdot 1$ & 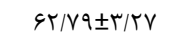 & $\Delta F / F T \pm T / T V$ & $G 9 / T V \pm T / T V$ & مصرف خوراى FF _ـ اروزگى (كرم) \\
\hline$\cdot 11$ & $1 / \Lambda r \pm \cdot / \cdot 9$ & $1 / 9 \mu \pm \cdot 1 \cdot 9$ & $1 / V V \pm \cdot 1 \cdot 9$ & ضريب تبديل غذايى FF- اروزگى \\
\hline.$/ 94$ & $\checkmark / \vee \vee \pm \cdot / \Delta \Delta$ & $V / \Delta T \pm \cdot / \Delta D$ & $V / / 9 \pm \pm \cdot / \Delta \Delta$ & وزن نسبى لاشه يوست كنده (درصد) \\
\hline.$/ 14$ & $r F / \kappa \cdot \pm \cdot / r \cdot$ & $r \varphi / \cdot \Lambda \pm \cdot / r \cdot$ & $r \mu / \Delta \Delta \pm \cdot / r \cdot$ & وزن نسبى ماهيجه سينهاى به وزن بدن (درصد) \\
\hline$\cdot 1 \cdot 1$ & $9 / 1 \cdot \pm \cdot 1 \cdot 0$ & S/TrE./.0 & $9 / \pi f \pm . / \cdot \Delta$ & 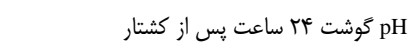 \\
\hline$<\cdot / \cdot 1$ & $r / 99 \pm \cdot / 19^{a}$ & $r / / \Delta \pm \cdot / r \cdot b$ & $r / \cdot V \pm \cdot / r \cdot b$ & آباندازى كَشت عَ ساعت پِ از كشتار (درصد) \\
\hline$\cdot / V \Delta$ & $\mid V / q T \pm Y / F r$ & $r \cdot / \& q \pm r / \Delta q$ & $\mid N / T r \pm T / \Delta S$ & קربى ماهيجه بر اساس ماده خشك (درصد) \\
\hline .1 .9 & $4 / 9 \vee \pm . / 11$ & 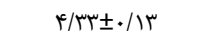 & $\tau / \& \Delta \pm . / 1$. & خاكستر بر اساس ماده خشك (درصد) \\
\hline \multirow[t]{2}{*}{.119} & $V / F \cdot \pm \cdot / \mathscr{F}$ & $\varepsilon / \& \& \pm \cdot / \Delta F$ & $\mathrm{~V} / \mathrm{M} \pm \cdot / \mp \Lambda$ & نيروى برش (نيوتن) \\
\hline & & & & رنخ كَشت بلافاصله پِ از كشتار \\
\hline$\cdot / \Delta \omega$ & $19 / V t \pm . / 99$ & $19 / r q \pm \cdot / V r$ & $10 / V T \pm \cdot / 99$ & قرمزى گَوشت (a*) \\
\hline$\cdot / N r$ & $r \cdot / v q \pm \cdot / v \cdot$ & $r \cdot / q \Lambda \pm \cdot / V V$ & $Y M / \Delta V \pm \cdot / V$. & زردى گوشت (b*) \\
\hline .19 & $\Delta V / \& Y \pm \cdot|\Lambda|$ & $\Delta V / \Lambda \cdot \pm \cdot / \wedge q$ & $\Delta N / Y| \pm \cdot| \Lambda \mid$ & روشنى گوشت (L) \\
\hline.$/ 9 \vee$ & $r g / A r \pm . / 9 \varphi$ & $r g / 9 \mid \pm . / V$. & $r \varepsilon / 1 \cdot \pm \cdot / q r$ & $\mathrm{C}$ \\
\hline \multirow[t]{3}{*}{.$/ F T$} & $\cdot / 19 \pm \cdot / \cdot r$ & $. / 91 \pm \cdot / \cdot r$ & $\cdot / 94 \pm . / \cdot r$ & $\mathrm{H}$ \\
\hline & T/T & 1/94 & & $\Delta \mathrm{D}$ \\
\hline & & & & رنخ كَوشت fr ساعت پِ از كشتار \\
\hline$\cdot 109$ & $\mid V / F \Delta \pm \cdot / \Delta V$ & $19 / \Delta V \pm \cdot / q T$ & $\mid \varepsilon / \Lambda F \pm \cdot / \Delta V$ & قرمزى گوشت (a*) \\
\hline 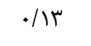 & $r \cdot / 99 \pm \cdot / \Delta \mathrm{V}$ & $r \cdot / r F \pm . / Q T$ & $r I / Q \Lambda \pm \cdot / \Delta V$ & زردى گوشت (b*) \\
\hline.$/ 49$ & $\Delta \Lambda / V \cdot \pm \cdot / \Lambda r$ & $\Delta N / T \Delta \pm \cdot / q$. & $\Delta Q / \Lambda \& \pm \cdot / \Lambda T$ & روشنى گوشت (L*) \\
\hline.$/ .4$ & $r \varepsilon / 99 \pm \cdot / r^{a b}$ & $\left\ulcorner\varepsilon / 19 \pm . / \% q^{b}\right.$ & $T V / A \cdot \pm \cdot / \Psi^{a}$ & $\mathrm{C}$ \\
\hline \multirow[t]{2}{*}{.$/ \Delta$} & $. / M \pm \pm . / . r$ & $\cdot / M \pm \cdot / \cdot r$ & $. / 9 T \pm . / .4$ & $\mathrm{H}$ \\
\hline & $1 / 90$ & $T / T V$ & & $\Delta \mathrm{D}$ \\
\hline
\end{tabular}


1. Abdolalizadeh Alvanegh, F., M. Ebrahimi and H. Daghigh Kia. 2017. Effect of in ovo injection of different L-arginine to L-lysine ratios on body growth, muscle production, and blood metabolite concentrations of day old Ross broilers Iranian Journal of Animal Science, 48: 207-217 (In Persian).

2. Al-Daraji, H.J., A.A. Al-Mashadani, W.K. Al-Hayani, A.S. Al-Hassani and H.A. Mirza. 2011. Influence of in ovo injection of L-arginine on productive and physiological performance of quail. Research Opinions in Animal and Veterinary Sciences, 1: 463-467.

3. Al-Daraji, H.J., A.A. Al-Mashadani, W.K. Al-Mashadani, A.S. Al-Hassani and H.A. Mirza. 2012. Effect of in ovo injection with L-arginine on productive and physiological traits of Japanese quail. South African Journal of Animal Science, 42: 139-145.

4. Allen, C.D., S.M. Russell and D.L. Fletcher. 1997. The Relationship of Broiler Breast Meat Color and $\mathrm{pH}$ to Shelf-Life and Odor Development. Poultry Science, 76: 1042-1046.

5. Ansari Pirsaraei, Z., A. Rahimi, H. Deldar, A.J. Sayyadi, M. Ebrahimi, A. Zareh Shahneh, M. Shivazad and M. Tebianian. 2018. Effect of feeding arginine on the growth performance, carcass traits, relative expression of lipogenic genes, and blood parameters of Arian broilers. Brazilian Journal of Poultry Science, 20: 363-370.

6. Ansari Pirsaraei, Z., M. Ebrahimi, A. Zare Shahneh, M. Shivazad and M. Tebianian. 2015. Determination of the best dietary level of L-arginine on improving growth performance, carcass traits and blood parameters in broiler chickens in the starter and grower periods. Research on Animal Production, 6: 87-95 (In Persian).

7. Association of Official Analytical Chemists (AOAC). 1984. Official Methods of Analysis. 14th edn. (Arlington, VA, Association of Official Analytical Chemists).

8. Ball, R.O., K.L. Urschel and P.B. Pencharz. 2007. Nutritional consequences of interspecies differences in arginine and lysine metabolism. The Journal of Nutrition, 137: 1626S-1641S.

9. Barbut, S. 1993. Colour measurements for evaluating the pale soft exudative (PSE) occurrence in turkey meat. Food Research International, 26: 39-43.

10. Berri, C., N. Wacrenier, N. Millet and E. Le Bihan-Duval. 2001. Effect of selection for body composition on muscle and meat characteristics of broilers from experimental lines. Poultry Science, 80: 833-838.

11. Chartrin, P., K. Meteau, H. Juin, M.D. Bernadet, G. Guy, C. Larzul, H. Remignon, J. Mourot, M.J. Duclos and E. Baéza. 2006. Effects of intramuscular fat levels on sensory characteristics of duck breast meat. Poultry Science, 85: 914-922.

12. Dransfeld, E. 1994. Modelling post-mortem tenderization-V: Inactivation of calpains. Meat Science, 37: 391-409.

13. Dransfield, E. and A.A. Sosnicki. 1999. Relationship between muscle growth and poultry meat quality. Poultry Science, 78: 743-746.

14. Ebrahimi, M., A. Zare Shahneh, M. Shivazad and Z. Ansari Pirsaraei. 2015. Evaluation of 24 days feeding L-arginin on performance, meat quality and blood metabolites in broilers. Animal Science Researches, 25: 61-72 (In Persian).

15. Ebrahimi, M., A. Zare Shahneh, M. Shivazad, Z. Ansari Pirsaraei and M. Ghafari Balesini. 2016. The effects of dietary L-arginine on some parameters of meat quality, intestine histology and immune system of 46-d old broiler chickens. Animal Science Researches, 26: 81-94 (In Persian).

16. Ebrahimi, M., A. Zare Shahneh, M. Shivazad, Z. Ansari Pirsaraei, M. Tebianian, C.A. Ruiz-Feria, M. Adibmoradi, K. Nourijelyani, F. Mohamadnejad. 2014a. The effect of feeding excess arginine on lipogenic gene expression and growth performance in broilers. British Poultry Science, 55: 81-88.

17. Ebrahimi, M., A. Zare Shahneh, M. Shivazad, Z. Ansari Pirsaraei, M. Tebianian, M. Adibmoradi, K. Nourijelyani. 2014b. The effects of dietary L-arginine on growth, meat production, and fat deposition in broiler chickens. Iranian Journal of Animal Science Researches, 5: 281-290 (In Persian).

18. Ebrahimi, M., F. Abdolalizadeh Alvanagh, M. Adibmoradi, H. Janmohammadi and Z. Rajabi. 2018. The impact of in ovo feeding with different $\mathrm{L}$ - arginine to $\mathrm{L}$ - lysine ratios on small intestine histological characteristics and immune system organs in day-old chicks. Animal Science Researches, 28: 177-191 (In Persian).

19. Edwards, N.M., N.D. Heberle and P.I. Hynd. 2016. The effect of in ovo administration of L-arginine on the hatchability and embryological development of broiler chicks. ASAP Animal Production 2016, Adelaide.

20. Fernandes, J.I.M., A.E. Murakami, E.N. Martins, M.I. Sakamoto and E.R.M. Garcia. 2009. Effect of arginine on the development of the pectoralis muscle and the diameter and the protein: deoxyribonucleic acid rate of its skeletal myofibers in broilers. Poultry Science, 88: 1399-1406.

21. Fletcher, D.L. 1999. Broiler breast meat color variation, pH, and texture. Poultry Science, 78: $1323-$ 1327.

22. Froning, G.W., A.S. Babji and F.B. Mather. 1978. The effect of pre-slaughter temperature, stress, struggle and anesthetization on color and textural characteristics of turkey muscle. Poultry Science, 57: 630-633. 
23. Gaafar, K.M., S.A. Selim and S.S. El-ballal. 2013. Effect of in-ovo administration with two levels of amino acids mixture on the performance of Muscovy ducks. Emirates Journal of Food and Agriculture, 25: 58-65.

24. Gao, T., M.M. Zhao, Y.J. Li, L. Zhang, J.L. Li, L.L. Yu, F. Gao and G.H. Zhou. 2017. Effects of in ovo feeding of L-arginine on the development of digestive organs, intestinal function and post-hatch performance of broiler embryos and hatchlings. Journal of Animal Physiology and Animal Nutrition, $1-10$.

25. Go, G.W., G. Wu and S.B. Smith. 2009. The growth performance, carcass traits, meat pH and color, and $\mathrm{CO} 2$ production in pigs supplementated arginine with conjugated linoleic acid. ASEB Journal, 23: 732-735.

26. Hurling, R., J.B. Rodell and H.D. Hunt. 1996. Fiber diameter and fish texture. Journal of Texture Studies, 27: 679-685.

27. Jiao, P., Y. Guo, X. Yang and F. Long. 2010. Effect of dietary arginine and methionine levels on broiler carcass traits and meat quality. Journal of Animal and Veterinary Advances, 9: 1546-1551.

28. Jobgen, W.S., S.K. Fried, W.J. Fu, C.J. Meininger and G. Wu. 2006. Regulatory role for the arginine-nitric oxide pathway in metabolism of energy substrates. The Journal of Nutritional Biochemistry, 17: 571-588.

29. Letsoalo, T.M.C. 2016. Effect of in ovo injection of glucose on egg hatchability, chick hatch-weight, productivity and carcass characteristics of indigenous Potchefstroom Koekoek chickens. Doctoral dissertation, University of Limpopo.

30. Ma, X., Y. Lin, Z. Jiang, C. Zheng, G. Zhou, D. Yu, T. Cao, J. Wang and F. Chen. 2010. Dietary arginine supplementation enhances antioxidative capacity and improves meat quality of finishing pigs. Amino Acids, 38: 95-102.

31. Maiorano, G., A. Sobolewska, D. Cianciullo, K. Walasik, G. Elminowska-Wenda, A. Sławińska, S. Tavaniello, J. ylińska, J. Bardowski and M. Bednarczyk. 2012. Influence of in ovo prebiotic and synbiotic administration on meat quality of broiler chickens. Poultry Science, 91: 2963-2969.

32. Munir, K., M.A. Muneer, E. Masaoud, A. Tiwari, A. Mahmud, R.M. Chaudhry and A. Rashid. 2009. Dietary arginine stimulates humoral and cell-mediated immunity in chickens vaccinated and challenged against hydropericardium syndrome virus. Poultry Science, 88: 1629-1638.

33. Piedrafita, J., R. Quintanilla, C. Sañudo, J.L. Olleta, M.M. Campo, B. Panea, G. Renand, F. Turin, S. Jabet, K. Osoro and M.C. Olivan. 2003. Carcass quality of 10 beef cattle breeds of the southwest of Europe in their typical production systems. Livestock Production Science, 82: 1-13.

34. Priolo, A., D. Micol and J. Agabriel. 2001. Effects of grass feeding systems on ruminant meat colour and flavour. A review. Animal Research, 50: 185-200.

35. Rey, C.R., A.A. Kraft, D.G. Topel, F.C. Parrish and D.K. Hotchkiss. 1976. Microbiology of pale, dark and normal pork. Journal of Food Science, 41: 111-116.

36. Saenmahayak, B., M. Singh, S.F. Bilgili and J.B. Hess. 2012. Influence of dietary supplementation with complexed zinc on meat quality and shelf life of broilers. International Journal of Poultry Science, 11: 28-32.

37. SAS Institute Inc. 2008. SAS/STAT User's Guide, Version 9.2. Cary, NC: SAS Institute Inc.

38. Schreurs, F.J.G., D. Van Der Heide, F.R. Leenstra and W. De Wit. 1995. Endogenous proteolytic enzymes in chicken muscles. Differences among strains with different growth rates and protein efficiencies. Poultry science, 74: 523-537.

39. Simões, J.A. 2006. Chemical and colour evaluation of meat from several Portuguese cattle breeds. Revista Portuguesa de Ciências Veterinárias, 559: 241.

40. Tan, B., Y. Yin, Z. Liu, X. Li, H. Xu, X. Kong, R. Huang, W. Tang, I. Shinzato, S.B. Smith and G. Wu. 2009. Dietary L-arginine supplementation increases muscle gain and reduces body fat mass in growing-finishing pigs. Amino Acids, 37: 169-175.

41. Warner, R.D., P.L. Greenwood, D.W. Pethick and D.M. Ferguson. 2010. Genetic and environmental effects on meat quality. Meat Science, 86: 171-183.

42. Wu, L.Y., Y.J. Fang and X.Y. Guo. 2011. Dietary L-arginine supplementation beneficially regulates body fat deposition of meat-type ducks. British Poultry Science, 52: 221-226.

43. Xu, Y.Q., Y.W. Guo, B.L. Shi, S.M. Yan and X.Y. Guo. 2018. Dietary arginine supplementation enhances the growth performance and immune status of broiler chickens. Livestock Science, 209: 813.

44. Zhang, H., K.E.C. Elliott, O.A. Durojaye, S.A. Fatemi, M.W. Schilling and E.D. Peebles. 2019. Effects of in ovo injection of L-ascorbic acid on growth performance, carcass composition, plasma antioxidant capacity and meat quality in broiler chickens. Poultry science, doi: 10.3382/ps/pez173.

45. Zhao, M.M., D.Q. Gong, T. Gao, L. Zhang, J.L. Li, P.A. Lv, L.L. Yu, F. Gao and G.H. Zhou. 2017. In ovo feeding of creatine pyruvate increases hatching weight, growth performance, and muscle growth but has no effect on meat quality in broiler chickens. Livestock Science, 206: 59-64. 


\title{
The Effect of in Ovo Injection with Different L-Arginine Levels on Hatchability, Growth, Performance and Meat Quality of Ross 308 Broiler Chickens
}

\section{Somayeh Omidi ${ }^{1}$, Marziyeh Ebrahimi ${ }^{2}$, Hossein Janmohammadi ${ }^{3}$, Hossein Taghipour ${ }^{4}$, Seyed Hadi Peighambardust ${ }^{5}$ and Hamidreza Ghassemzadeh ${ }^{6}$}

1- M.Sc. Student of Department of Animal Science, Faculty of Agriculture, University of Tabriz, Iran

2- Assistant professor of Department of Animal Science, Faculty of Agriculture, University of Tabriz, Tabriz, Iran, (Corresponding author: mailto:marzebrahimi@tabrizu.ac.ir)

3- Professor of Department of Animal Science, Faculty of Agriculture, University of Tabriz, Tabriz, Iran

4- M.Sc. of East Azerbaijan Institute of Research and Education of Agriculture and Natural Resources, Tabriz, Iran

5- Professor of Department of Feed Science and Technology, Faculty of Agriculture, University of Tabriz, Tabriz, Iran

6- Professor of Department of Biosystem Engineering, Faculty of Agriculture, University of Tabriz, Tabriz, Iran Received: May 18, $2019 \quad$ Accepted: June 2, 2019

\begin{abstract}
Previous studies have indicated the positive impact of dietary supplementation of arginine on meat quality and growth, though there is no report regarding the effect of in ovo injection of arginine on meat quality. So, the aim of this study was to evaluate the effect of in ovo injection of different levels of L-arginine on hatchability, growth performance, and meat quality of Ross 308 broiler chickens. In this research, 300 fertile eggs were used in a completely randomized design with three experimental treatments (levels of 0.5 and $1 \%$ of L- arginine and total control). Experimental groups included: 1) in ovo injection of $0.5 \% \mathrm{~L}$-arginine, 2- in ovo injection of $1 \%$ L-arginine, 3) total control group \{collected control 1 (injection of distilled water) and control 2 (no injection)\}, which were injected on d 14 of incubation. After hatching, broiler chicks of arginine treatments and total control group were divided each into four equal groups and placed randomly into four cages (replicates of the experiment). The rearing period continued from hatching to $\mathrm{d} 24$. In order to investigate the quality of meat, on day 24 , three chicks from each replicate (12 chicks per each treatment) were weighed, slaughtered and their breast muscles were used for meat quality evaluation. Based on the results, in ovo injection of different levels of L- arginine did not significantly affect 1, 10 and 24 day-old birds' weight, daily weight gain, feed intake, feed conversion ratio, and relative weight of scalped carcass and breast muscle $(\mathrm{P}>0.05)$. Although, meat $\mathrm{pH}$, meat fat and Ash content, and meat color indexes $\left(\mathrm{a}^{*}, \mathrm{~b}^{*}, \mathrm{~L}^{*}, \mathrm{H}\right)$ were not affected by $\mathrm{L}-$ arginine in ovo injection treatments $(\mathrm{P}>0.05)$, meat water loss, $\mathrm{D}$, and $\mathrm{C}$ indexes were significantly affected by experimental treatments $\left(\begin{array}{ll}\mathrm{P} & 0.05\end{array}\right)$. Based on the overall results of this study, in ovo injection with different levels of L- arginine could not have a significantly noticeable effect on growth performance, meat production and/or meat quality of 24 day- old broiler chickens.
\end{abstract}

Keywords: Broiler chickens, In ovo injection, L- arginine, Meat quality 